\title{
Commentary: "Brain-Doping," Is It a Real Threat?
}

\author{
Zhiqiang Zhu ${ }^{1,2}$, Junhong Zhou ${ }^{3,4}$, Brad Manor ${ }^{3,4}, X_{i}$ Wang $^{1}$, Weijie Fu ${ }^{1 *}$ and Yu Liu ${ }^{1 *}$ \\ ${ }^{1}$ School of Kinesiology, Shanghai University of Sport, Shanghai, China, ${ }^{2}$ School of Physical Education, East China Jiao Tong \\ University, Nanchang, China, ${ }^{3}$ Hinda and Arthur Marcus Institute for Aging Research, Hebrew SeniorLife, Boston, MA, \\ United States, ${ }^{4}$ Harvard Medical School, Boston, MA, United States
}

Keywords: tDCS, sport/exercise performance, methodological hurdles, neuro-physiological mechanism, inter-personal differences, brain structure and function

\section{A Commentary on}

"Brain-Doping," Is It a Real Threat?

by Holgado, D., Vadillo, M. A., and Sanabria, D. (2019). Front. Physiol. 10:483. doi: $10.3389 /$ fphys.2019.00483

\section{OPEN ACCESS}

Edited by:

Giovanni Messina,

University of Foggia, Italy

Reviewed by:

Samuele Maria Marcora University of Kent, United Kingdom

Roberto Codella,

University of Milan, Italy

${ }^{*}$ Correspondence:

Weijie Fu

fuweijie@sus.edu.cn; fuweijie315@163.com

Yu Liu

yuliu@sus.edu.cn

Specialty section:

This article was submitted to

Exercise Physiology,

a section of the journal

Frontiers in Physiology

Received: 04 July 2019 Accepted: 21 November 2019 Published: 05 December 2019

Citation:

Zhu Z, Zhou J, Manor B, Wang X, Fu W and Liu Y (2019) Commentary: "Brain-Doping," Is It a Real Threat?

Front. Physiol. 10:1489

doi: 10.3389/fphys.2019.01489

\section{INTRODUCTION}

On the basis of several recent meta-analyses synthesizing the effects of transcranial direct current stimulation (tDCS) on sport performance (Lattari et al., 2018; Machado et al., 2018; Holgado et al., 2019a), Holgado et al. (2019b) concluded that there is insufficient evidence to support an ergogenic or "brain-doping" effect of tDCS on sport and/or physical performance. The authors also highlighted that the exact neuro-modulatory mechanisms through which tDCS may improve human performance remain largely unclear. We describe here more explicitly several important limitations of the majority of tDCS research to date. We also examine potential mechanisms of action, and provide recommendations that we believe are needed to more robustly study the ergogenic effects of tDCS moving forward.

\section{OVERCOMING METHODOLOGICAL "HURDLES" IN tDCS RESEARCH}

Inconsistencies in tDCS effectiveness both within and between studies has been well-documented (Lattari et al., 2018; Machado et al., 2018; Holgado et al., 2019b) and likely arises from the combination of multiple issues. Across studies, numerous different devices have been utilized to administer stimulation, which may vary considerably in the current flow properties that they produce (Hahn et al., 2013; Zhang and Li, 2015). The characteristics of tDCS intervention, including the cortical target of interest, electrode size, electrode materials, and the intensity of current flow, have also varied considerably between studies, and moreover, are often insufficiently reported (Palm et al., 2014; Kouzani et al., 2016; Machado et al., 2018; Holgado et al., 2019a). Complicating matters further is that across subjects within studies, the same tDCS intervention may produce very different electrical fields in the cortex, and have different effects on brain function, due to inter-subject variation in both anatomy and physiology (Wiethoff et al., 2014; Li et al., 2015; Sanchez-Kuhn et al., 2018). Together, these challenges highlight the need for standardized reporting of tDCS, as well as the application of advanced technology to help measure and even "personalize" current flow. 
Fortunately, recent technological advances offer promise to help researchers estimate the electric fields induced by tDCS, customize montages to individual head and brain anatomy, and examine the effects of tDCS on brain physiology. For example, Laakso et al. (2016) used a finite element modeling technique on a standardized brain template to determine the characteristics of the electrical field generated by tDCS. Such a technique now offers a data driven approach to developing tDCS montages that more likely target the cortical region of interest. Applying such a technique to individual brain MRIs also promises to help establish dose-response relationships between tDCS-induced electric fields and potential changes in functional performance. Beyond this, we believe that researchers should consider administering tDCS via an array of smaller electrodes, and in appropriate circumstances using montages created via modeling-based optimization techniques (Ruffini et al., 2014), to create more focal and "personalized" targets of tDCS (Madhavan and Stinear, 2010; Bikson et al., 2012; Li et al., 2015; Opitz et al., 2015). Together, we believe that the combination of these new techniques will ultimately help to produce larger, more consistent effects of tDCS intervention.

\section{SPORT PERFORMANCE IS MORE THAN STRENGTH AND ENDURANCE}

Several published meta-analyses have combined data from studies on the effects of tDCS on both muscle strength and endurance (Machado et al., 2018; Holgado et al., 2019a). These two muscle functions stem from distinct neuro-physiologic procedures, are likely to be differentially affected by cortical neuromodulation, and should be considered separately in such analyses. Furthermore, numerous factors beyond muscle strength and endurance contribute to sports performance (Miller and Clapp, 2011; Loprinzi et al., 2013). Such factors as sensory perception and processing, fatigue, perceived exertion, multiple aspects of executive function including dual tasking and response inhibition, and neural recovery depend upon supraspinal function and appear to be modifiable via tDCS (Zhou et al., 2014; Wang et al., 2015; Vecchio et al., 2018; Angius et al., 2019) A better understanding of the acute and longer-term impact of tDCS on these factors is needed before definitive neuro-doping claims should be made.

\section{ONE POTENTIAL MECHANISM OF ACTION}

Most studies to date have focused on the effects of tDCS on functional performance; e.g., tDCS targeting the bilateral motor cortex has been shown to improved cycling performance in healthy adults (Angius et al., 2018). Much more work is thus needed to uncover the neurophysiological mechanisms through which tDCS may improve such performance. Understanding this, in turn, will enable the development of neuromodulatory interventions directly aimed at enhancing such mechanisms.

Several potential mechanisms of action through which tDCS alters functional performance have been described. Such efforts have focused on the effects of tDCS on neurochemical transmitters (e.g., GABA, dopamine, adenosine) (Kuo et al., 2008; McLaren et al., 2018), as well as neurophysiologic responses (Labruna et al., 2019). GABA, for example, is one of the most important inhibitory neurotransmitters in the brain and has been linked to motor performance (Krause et al., 2013; Kolasinski et al., 2019). To this end, Kim et al. (2014) demonstrated that compared to cathodal or sham stimulation, anodal tDCS targeting the hand area of the left primary motor cortex induced a reduction in GABA concentration within this brain region. Excitingly, those who experienced a greater decrease in GABA concentration exhibited greater improvements in performance on both motor learning and motor memory tasks.

Additional work is also needed to better understand the links between $\mathrm{tDCS}$-induced changes in neurophysiology and function outcomes. Labruna et al. (2019) recently published a promising study, for example, that used single-pulse transcranial magnetic stimulation (TMS) over the primary motor cortex to measure the effects of tDCS on resting motor thresholds (rMT). Results suggested that following tDCS, participants who exhibited a greater reduction in rMT tended to improve their performance more in a motor learning task. Moving forward, more studies such as these that combine both functional and mechanistic outcomes will help us better understand and optimize the effects of tDCS on sport performance.

\section{IS tDCS A “NEURO-DOPING” THREAT?}

According to the current World Anti-Doping Code (WADA, 2019), a substance or method should be considered as "doping" if it meets two of three criteria based upon scientific criteria and/or experience: (1) it has potential beneficial effects on athletic performance, (2) it poses potential health risks to athletes, and (3) it violates the spirit of sport. We believe that tDCS has strong potential to enhance athletic performance, especially as advances in technology, modeling, and methodology help overcome the many challenges of traditional tDCS research. At the same time, tDCS is widely believed to pose non-significant risk to participants when recommended procedures are followed (Brunoni et al., 2011; Bikson et al., 2016; Jackson et al., 2017). tDCS therefore holds strong promise to improve performance without posing significant health risks to athletes. As such, we believe that determination of tDCS as a neuro-doping strategy will ultimately come down to the challenging ethical question of whether or not it negatively impacts the spirit of sport and fair competition.

\section{AUTHOR CONTRIBUTIONS}

ZZ, JZ, BM, XW, WF, and YL conceived, drafted, and revised the manuscript. All authors read and approved the final manuscript.

\section{FUNDING}

This work was supported by the National Natural Science Foundation of China (11932013); National Key Research and Development Program of China (2018YFF0300500). 


\section{REFERENCES}

Angius, L., Mauger, A. R., Hopker, J., Pascual-Leone, A., Santarnecchi, E., and Marcora, S. M. (2018). Bilateral extracephalic transcranial direct current stimulation improves endurance performance in healthy individuals. Brain Stimul. 11, 108-117. doi: 10.1016/j.brs.2017.09.017

Angius, L., Santarnecchi, E., Pascual-Leone, A., and Marcora, S. M. (2019). Transcranial direct current stimulation over the left dorsolateral prefrontal cortex improves inhibitory control and endurance performance in healthy individuals. Neuroscience 419, 34-45. doi: 10.1016/j.neuroscience.2019.08.052

Bikson, M., Grossman, P., Thomas, C., Zannou, A. L., Jiang, J., Adnan, T., et al. (2016). Safety of transcranial direct current stimulation: evidence based update 2016. Brain Stimul. 9, 641-661. doi: 10.1016/j.brs.2016.06.004

Bikson, M., Rahman, A., Datta, A., Fregni, F., and Merabet, L. (2012). High-resolution modeling assisted design of customized and individualized transcranial direct current stimulation protocols. Neuromodulation 15, 306-315. doi: 10.1111/j.1525-1403.2012.00481.x

Brunoni, A. R., Fregni, F., and Pagano, R. L. (2011). Translational research in transcranial direct current stimulation (tDCS): a systematic review of studies in animals. Rev. Neurosci. 22, 471-481. doi: 10.1515/RNS.2011.042

Hahn, C., Rice, J., Macuff, S., Minhas, P., Rahman, A., and Bikson, M. (2013). Methods for extra-low voltage transcranial direct current stimulation: current and time dependent impedance decreases. Clin. Neurophysiol. 124, 551-556. doi: 10.1016/j.clinph.2012.07.028

Holgado, D., Vadillo, M. A., and Sanabria, D. (2019a). The effects of transcranial direct current stimulation on objective and subjective indexes of exercise performance: a systematic review and meta-analysis. Brain Stimul. 12, 242-250. doi: 10.1016/j.brs.2018.12.002

Holgado, D., Vadillo, M. A., and Sanabria, D. (2019b). 'Brain-Doping', Is it a real threat? Front. Physiol. 10:483. doi: 10.3389/fphys.2019.00483

Jackson, M. P., Bikson, M., Liebetanz, D., and Nitsche, M. (2017). How to consider animal data in tDCS safety standards. Brain Stimul. 10, 1141-1142. doi: 10.1016/j.brs.2017.08.004

Kim, S., Stephenson, M. C., Morris, P. G., and Jackson, S. R. (2014). tDCS-induced alterations in GABA concentration within primary motor cortex predict motor learning and motor memory: a $7 \mathrm{~T}$ magnetic resonance spectroscopy study. Neuroimage 99, 237-243. doi: 10.1016/j.neuroimage.2014.05.070

Kolasinski, J., Hinson, E. L., Zand, A. P. D., Rizov, A., Emir, U. E., and Stagg, C. J. (2019). The dynamics of cortical GABA in human motor learning. J. Physiol. 597, 271-282. doi: 10.1113/jp276626

Kouzani, A. Z., Jaberzadeh, S., Zoghi, M., Usma, C., and Parastarfeizabadi, M. (2016). Development and validation of a miniature programmable tDCS device. IEEE Trans. Neural Syst. Rehabil. Eng. 24, 192-198. doi: $10.1109 /$ tnsre.2015.2468579

Krause, B., Marquez-Ruiz, J., and Kadosh, R. C. (2013). The effect of transcranial direct current stimulation: a role for cortical excitation/inhibition balance? Front. Hum. Neurosci. 7:602. doi: 10.3389/fnhum.2013.00602

Kuo, M. F., Paulus, W., and Nitsche, M. A. (2008). Boosting focally-induced brain plasticity by dopamine. Cereb. Cortex 18, 648-651. doi: 10.1093/cercor/bhm098

Laakso, I., Tanaka, S., Mikkonen, M., Koyama, S., Sadato, N., and Hirata, A. (2016). Electric fields of motor and frontal tDCS in a standard brain space: a computer simulation study. Neuroimage 137, 140-151. doi: 10.1016/j.neuroimage.2016.05.032

Labruna, L., Stark-Inbar, A., Breska, A., Dabit, M., Vanderschelden, B., Nitsche, M. A., et al. (2019). Individual differences in TMS sensitivity influence the efficacy of tDCS in facilitating sensorimotor adaptation. Brain Stimul. 12, 992-1000. doi: 10.1016/j.brs.2019.03.008

Lattari, E., Oliveira, B. R., Junior, R. S. M., Neto, S. R. M., Oliveira, A. J., Neto, G. A. M., et al. (2018). Acute effects of single dose transcranial direct current stimulation on muscle strength: a systematic review and meta-analysis. PLoS ONE 13:e0209513. doi: 10.1371/journal.pone. 0209513

Li, L. M., Uehara, K., and Hanakawa, T. (2015). The contribution of interindividual factors to variability of response in transcranial direct current stimulation studies. Front. Cell. Neurosci. 9:181. doi: 10.3389/fncel.2015.00181
Loprinzi, P. D., Herod, S. M., Cardinal, B. J., and Noakes, T. D. (2013). Physical activity and the brain: a review of this dynamic, bi-directional relationship. Brain Res. 1539, 95-104. doi: 10.1016/j.brainres.2013. 10.004

Machado, D. G. D. S., Unal, G., Andrade, S. M., Moreira, A., Altimari, L. R., Brunoni, A. R., et al. (2018). Effect of transcranial direct current stimulation on exercise performance: a systematic review and meta-analysis. Brain Stimul. 12, 593-605. doi: 10.1016/j.brs.2018.12.227

Madhavan, S., and Stinear, J. W. (2010). Focal and bidirectional modulation of lower limb motor cortex using anodal transcranial direct current stimulation. Brain Stimul. 3, 42-50. doi: 10.1016/j.brs.2009.06.005

McLaren, M. E., Nissim, N. R., and Woods, A. J. (2018). The effects of medication use in transcranial direct current stimulation: a brief review. Brain Stimul. 11, 52-58. doi: 10.1016/j.brs.2017.10.006

Miller, B. T., and Clapp, W. C. (2011). From vision to decision: the role of visual attention in elite sports performance. Eye Contact Lens 37, 131-139. doi: 10.1097/ICL.0b013e3182190b7f

Opitz, A., Paulus, W., Will, S., Antunes, A., and Thielscher, A. (2015). Determinants of the electric field during transcranial direct current stimulation. Neuroimage 109, 140-150. doi: 10.1016/j.neuroimage.2015.01.033

Palm, U., Feichtner, K. B., Hasan, A., Gauglitz, G., Langguth, B., Nitsche, M. A., et al. (2014). The role of contact media at the skin-electrode interface during transcranial direct current stimulation (tDCS). Brain Stimul. 7, 762-764. doi: 10.1016/j.brs.2014.06.006

Ruffini, G., Fox, M. D., Ripolles, O., Miranda, P. C., and Pascual-Leone, A. (2014). Optimization of multifocal transcranial current stimulation for weighted cortical pattern targeting from realistic modeling of electric fields. Neuroimage 89, 216-225. doi: 10.1016/j.neuroimage.2013.12.002

Sanchez-Kuhn, A., Perez-Fernandez, C., Moreno, M., Flores, P., and SanchezSanted, F. (2018). Differential effects of transcranial direct current stimulation (tDCS) depending on previous musical training. Front. Physiol. 9:1465. doi: $10.3389 /$ fpsyg. 2018.01465

Vecchio, F., Di Iorio, R., Miraglia, F., Granata, G., Romanello, R., Bramanti, P., et al. (2018). Transcranial direct current stimulation generates a transient increase of small-world in brain connectivity: an EEG graph theoretical analysis. Exp. Brain Res. 236, 1117-1127. doi: 10.1007/s00221-018$5200-\mathrm{z}$

WADA (2019). World Anti-Doping Code 2015 with 2019 Amendments, 4.3 C.F.R.

Wang, Y., Hao, Y., Zhou, J. H., Fried, P. J., Wang, X. Y., Zhang, J., et al. (2015). Direct current stimulation over the human sensorimotor cortex modulates the brain's hemodynamic response to tactile stimulation. Eur. J. Neurosci. 42, 1933-1940. doi: 10.1111/ejn.12953

Wiethoff, S., Hamada, M., and Rothwell, J. C. (2014). Variability in response to transcranial direct current stimulation of the motor cortex. Brain Stimul. 7, 468-475. doi: 10.1016/j.brs.2014.02.003

Zhang, Y., and Li, Z. H. (2015). Improving the accuracy of time-difference measurement by reducing the impact of baseline shift. IEEE Trans. Instrum. Meas. 64, 3013-3020. doi: 10.1109/tim.2015.2437635

Zhou, J. H., Hao, Y., Wang, Y., Jor'dan, A., Pascual-Leone, A., Zhang, J., et al. (2014). Transcranial direct current stimulation reduces the cost of performing a cognitive task on gait and postural control. Eur. J. Neurosci. 39, 1343-1348. doi: 10.1111/ejn.12492

Conflict of Interest: The authors declare that the research was conducted in the absence of any commercial or financial relationships that could be construed as a potential conflict of interest.

Copyright (๑) 2019 Zhu, Zhou, Manor, Wang, Fu and Liu. This is an open-access article distributed under the terms of the Creative Commons Attribution License (CC $B Y)$. The use, distribution or reproduction in other forums is permitted, provided the original author(s) and the copyright owner(s) are credited and that the original publication in this journal is cited, in accordance with accepted academic practice. No use, distribution or reproduction is permitted which does not comply with these terms. 